\title{
Solar flare detection system based on global positioning system data: First results
}

\author{
A. García-Rigo *, M. Hernández-Pajares, J.M. Juan, J. Sanz \\ Research Group of Astronomy and Geomatics, Technical University of Catalonia (gAGE/UPC), Mod.C3 Campus Nord UPC, \\ c/Jordi Girona 1-3, E08034 Barcelona, Spain
}

Received 12 January 2006; received in revised form 17 September 2006; accepted 18 September 2006

\begin{abstract}
A solar flare detector has been developed using the Global Positioning System (GPS) capabilities to monitor the ionospheric Total Electron Content (TEC). It is based on obtaining the variation of the vertical TEC data with respect to the previous sidereal day and then, on performing a second order time difference to obtain the information of the instantaneous TEC changes. The detector can be applied looking for solar flares backwards or in real time as well. To make a first assessment of the solar flare detector, several specific days, when there is the certainty that a solar flare had occurred, have been analyzed. The results, which are presented in this work, have been obtained from the X17.2 flare on 28th October, 2003, the X5.7 flare on 14th July, 2000 and the X7.1 flare on 20th January, 2005, using the GPS data of the International GNSS Service (IGS) network with a sampling rate of $30 \mathrm{~s}$. The results obtained are compatible with the results presented previously by different authors, and with the flare records included in the Geostationary Operational Environmental Satellite (GOES) database. In addition, several receivers, located in the southern hemisphere and far from the geomagnetic equator, detect a lower overionization amplitude than other receivers with similar mean solar zenith angle. Moreover, minor disturbances in the TEC enhancement can also be observable and studied.
\end{abstract}

(c) 2006 COSPAR. Published by Elsevier Ltd. All rights reserved.

Keywords: Solar flares; Global positioning system; Ionosphere; Total electron content

\section{Introduction}

Solar flares are one of the most violent events that take place on the Sun's surface. They are generated near sunspots and are characterized by the emission of radiation in all electromagnetic ranges and by the ejection of charged particles. The radiation produced by a solar flare is especially important in the ultraviolet and X-ray bands and reaches the Earth in about $8 \mathrm{~min}$. On the contrary, the particles ejected (a mix of electrons, protons and heavy nuclei) take 1 or 2 days to arrive, following the Interplanetary Magnetic Field (IMF) lines. Therefore, radiation reaches the Earth before particles. Moreover, the high

\footnotetext{
* Corresponding author.

E-mail address: agarcia@ma4.upc.edu (A. García-Rigo, M. Hernández-Pajares, J.M. Juan, J. Sanz).
}

energy related to this radiation produces a sudden increase of ionization in our atmosphere, and thus a sudden increase of the Total Electron Content (Mendillo et al., 1974). As the ionization is mainly produced in the ionosphere, a way to detect solar flares is by monitoring the TEC of this part of the atmosphere.

There are several systems that detect solar flares. Some are direct systems, such as the meteorological satellite GOES (Geostationary Operational Environmental Satellite) that measures the X-rays coming from the Sun. Others are indirect systems, such as the Global Positioning System, which is based on a satellite constellation of more than 24 dual frequency transmitters in L-band. This system allows to analyze the ionospheric response to certain major solar flares with a high simultaneous temporal and spatial resolution. In this context, the main goal of this work is to illustrate how the GPS system can be used to monitor 
the electron content variations induced by solar flares. This can be done globally, automatically and for long periods of time, and in an easy and efficient way.

\section{Description of the technique}

First of all, the dual frequency GPS data is processed in order to obtain the carrier phase ionospheric combination $L_{\mathrm{I}}$ and the code ionospheric combination $P_{\mathrm{I}}$ in which the geometry, clocks and troposphere dependence have been cancelled out (see Eq. (1), both in length units). These combinations provide the Total Electron Content (TEC), which contains, in particular, the ionization enhancement expected during solar flares (see, for instance, Hernández-Pajares et al., 2002). Indeed:

$L_{\mathrm{I}}=S T E C+B_{\mathrm{I}}$

$P_{\mathrm{I}}=S T E C+D C B$,

where STEC is the slant TEC (i.e., the TEC in the line of sight of the GPS ray path), $B_{\mathrm{I}}$ the phase ambiguity and $D C B$ the Delay Code Bias. In addition, it is important to emphasize that the code is much noisier than the carrier phase. Therefore, it is much more precise to work with $L_{\mathrm{I}}$ measurements, though the phase ambiguity has to be solved.

Afterwards, the strategy used is the one proposed by Hernández-Pajares et al. (1997) which consists of temporal differences between consecutive sidereal days, which can provide the amplitude of the TEC variation. Indeed, as the ionospheric ionization in normal conditions (i.e., with low geomagnetic disturbances) varies slowly and is quite similar from 1 day to another, periodic effects such as the ionospheric diurnal and seasonal variations are mostly cancelled. On the contrary, sporadic unexpected effects such as the overionization related to a solar flare remain. In this way, the variations of the ionospheric combinations with respect to the previous sidereal day, i.e., approximately $23 \mathrm{~h} 56 \mathrm{~m}$ before, have been calculated in this work and they will be called $\delta L_{\mathrm{I}}$ and $\delta P_{\mathrm{I}}$ from now on. In addition, the sidereal day variation of the slant TEC (called $\delta S T E C$ ) can be obtained by rearranging Eq. (1) as follows:

$\delta L_{\mathrm{I}}=\delta S T E C+\delta B_{\mathrm{I}}$

$\delta P_{\mathrm{I}}=\delta S T E C+\delta D C B$

where $\delta D C B \simeq 0$ due to the high repeatabilities of the instrumental delay code biases between consecutive days, and specially at similar local times.

Then, the bias $\delta B_{\mathrm{I}}$ can be estimated (from Eq. (2)) by applying an alignment of $\delta L_{\mathrm{I}}$ with $\delta P_{\mathrm{I}}$, by averaging its difference within the same continuous combined carrier phase arch (i.e., no cycle slips affecting both carrier phases, $L_{\mathrm{I}}(t)$ and $\left.L_{I}(t-86,160)\right)$, as written in Eq. (3). It must be taken into account that this combined arch should have a minimum number of samples to avoid an excessively deviated average (for instance, 30 samples in this work). $\hat{\delta B_{\mathrm{I}}}=\left\langle\delta L_{\mathrm{I}}-\delta P_{\mathrm{I}}\right\rangle_{\text {combined arch }}$.

In order to cancel the dependence on the GPS ray inclination, the $\delta S T E C$ values are transformed into the sidereal day difference of vertical TEC (i.e., in the radial direction regarding to the Earth geocenter) values (from now on referred to as $\delta V$, where $\delta$ stands for the sidereal day difference). In this work, this is done using the mapping function considering a thin single layer model of the ionosphere at a height of $450 \mathrm{~km}$, but considering an elevation mask of $30^{\circ}$ to minimize mapping errors.

The following step is to apply a second order time difference of $\delta V$ between a pair of samples separated by a short period of time (in our case, $360 \mathrm{~s}$ ), which will be referred to as $\mathrm{d}^{2} \delta V$. Therefore, the remaining low frequency TEC variations are detrended in a simple way and, consequently, the information of the instantaneous changes contained in $\delta V$ is obtained (similarly as with the rate of TEC change or rTEC in Liu et al. (2004)). For that purpose, the first order and second order time difference of $\delta V\left(\mathrm{~d} \delta V\right.$ and $\mathrm{d}^{2} \delta V$, respectively) are computed as follows:

$\delta V(t)=V(t)-V(t-86160)$
$\mathrm{d} \delta V(t)=\delta V(t)-\delta V(t-360)$
$\mathrm{d}^{2} \delta V(t)=\mathrm{d} \delta V(t)-\mathrm{d} \delta V(t-360)$,

where 86,160 is the approximate number of seconds of one sidereal day.

Afterwards, an analysis of the $\mathrm{d}^{2} \delta V$ amplitude values is performed, searching for the typical overionization pattern (in $\mathrm{d}^{2} \delta V$, an increase is followed by a symmetrical decrease, as shown for instance in Fig. 1), in order to detect solar flares pointing to the Earth. Additionally, it is necessary to discard similar patterns induced by other effects such as the Travelling Ionospheric Disturbances (TIDs) or the Scintillations. Taking into account that the solar radiation simultaneously affects the whole sunlit area of the Earth and that the other phenomena affect only local regions, the following conditions must be fulfilled to avoid false detections:

(1) Signals of many satellites of one receiver must have a simultaneous TEC increase and with similar amplitudes (Signals of all satellites in view of a given receiver above a certain elevation mask, $30^{\circ}$ in our case).

(2) Signals of many receivers in the sunlit region must have this significant TEC increase at almost the same time but with a significant change of amplitude (in case the flare is powerful enough, it should be for all receivers).

If just one of those conditions is not fulfilled, it is considered that the overionization cannot be due to a solar flare. The results presented in this work have been obtained considering a minimum number of three satellites to fulfill the first condition and a minimum number of five receivers 

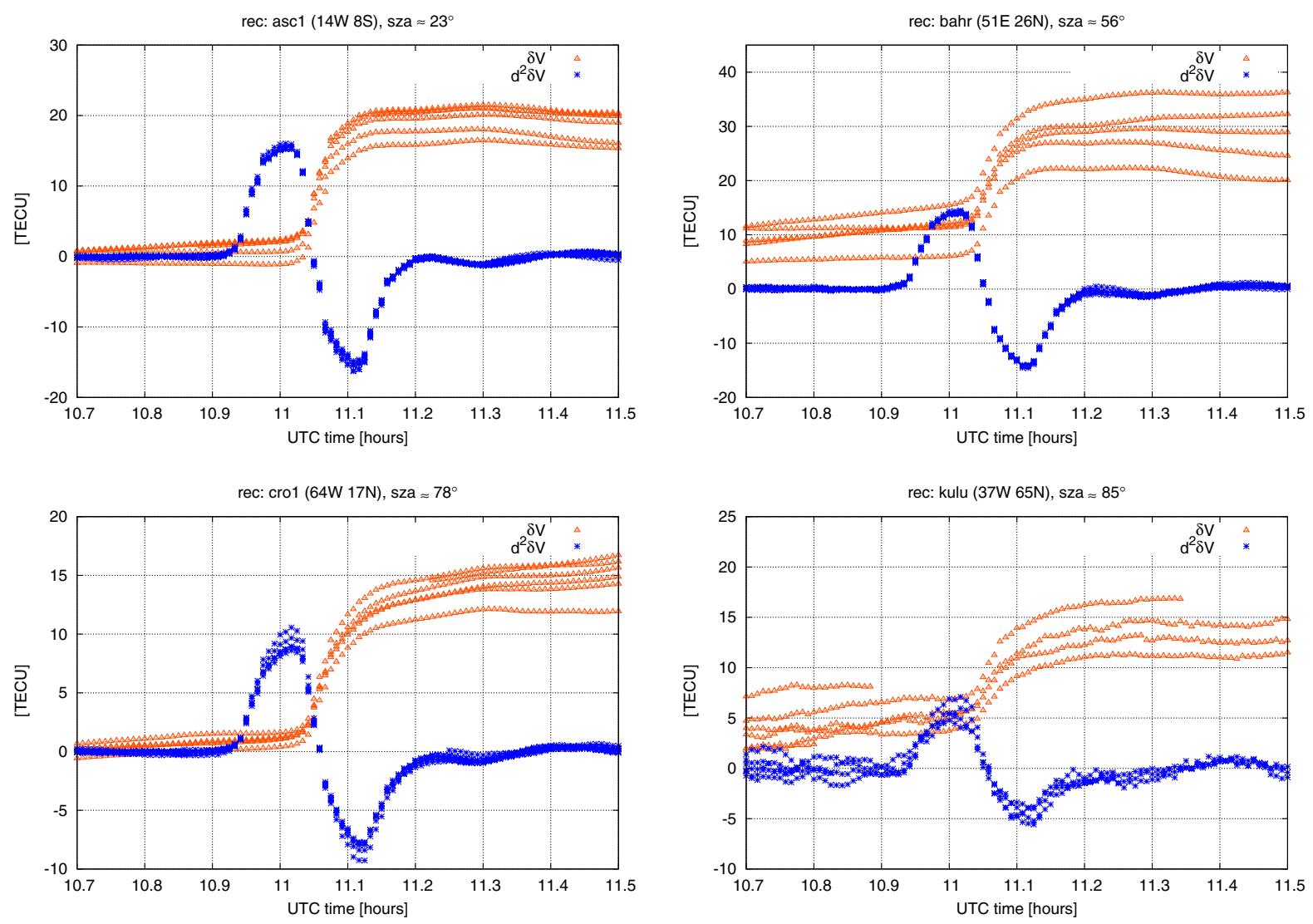

Fig. 1. $\delta V$ and $\mathrm{d}^{2} \delta V$ as function of time obtained from the GPS signals gathered by the receivers ascl (upper left panel), bahr (upper right panel), crol (lower left panel) and kulu (lower right panel) during the flare on 28th October, 2003, and considering a masking angle of $30^{\circ}$. The longitude, latitude and mean solar zenith angle of each station are indicated in the corresponding panel.

widely distributed to fulfill the second condition (and thus confirm flare detection). In addition, it is important to emphasize that the amplitude of the flare signal changes between different satellites of one receiver due to the solar zenith angle dependence of the overionization. Furthermore, this effect is more significant when comparing different receivers in different geographical locations (see Fig. 3).

The method that has been employed to implement the detector is firstly based on five different states to monitor the $\mathrm{d}^{2} \delta V$ data. These states are: start, up, fixedup, fixeddown and TEC enhancement. In normal conditions, i.e., with low geomagnetic disturbances, the state is the one labelled as start. The detector gets the amplitude of $\mathrm{d}^{2} \delta V$ sample by sample and then three possibilities are distinguished: the amplitude is higher than a threshold of detection, it is lower than minus this threshold of detection or it is in between. According to these possibilities and the last state for the satellite in consideration, the decisions and transitions between states are different. In this way, when an overionization pattern happens, the detector finds a TEC increase greater than the threshold (state up), identifies the peak of the increase in $\mathrm{d}^{2} \delta V$ (state fixedup), finds a symmetrical decrease (state down) and then identifies the lowest sample of the decrease (state fixeddown). If this order of state transitions is found in almost all GPS signals in the sunlit region, satisfying the previously mentioned conditions, we assume that a solar flare has happened and has been detected.

The detector can also be based on a more general criteria. In this case, only the positive TEC increase is searched in the $\mathrm{d}^{2} \delta V$ data. The main interest of this consideration will be shown in Section 3 .

It is worth mentioning that the threshold of detectability in $\mathrm{d}^{2} \delta V$ has been fixed in 0.2 TEC units or TECUs ( 1 TECU is equivalent to $10^{16} \mathrm{el} / \mathrm{m}^{2}$ ) taking into account two considerations. The first one is that more flares can be detected if this threshold is low. The second one is that fewer false detections can occur if this threshold is high. Consequently, it has been necessary to establish a compromise between both considerations.

\section{Computations and results}

The computations to detect and analyze solar flares are made under an standard PC running LINUX operating system using the $30 \mathrm{~s}$ GPS data of at least 90 stations of the International GNSS Service (IGS) network. These stations are selected regularly all over the world for every day computed, considering that only one available station is selected for each cell of $20^{\circ}$ in longitude range and $15^{\circ}$ in 
latitude range. That allows the observation of the ionospheric response with a high spatial coverage whenever a solar flare pointing to the Earth happens.

To make a first assessment of the solar flare detector, several specific days, when there is the certainty that a solar flare had occurred, have been analyzed. In particular, the ones occurred on 28th October, 2003, on 14th July, 2000 and on 20th January, 2005.

First of all, the flare occurring on 28th October, 2003 (also referred to as the Halloween storm) has been analyzed in detail. It was a really powerful flare (it is classified as X17.2) that occurred at the center of the solar disc with the peak of intensity at about 11 UT.

To detect this solar flare, 95 available GPS permanent receivers have been selected all over the world. After applying the technique developed in this work, the results show that an overionization at 11 UT is detected on 33 receivers on that day, with a peak of intensity in $\delta V$ that reached more than 15 TECUs near the maximum solar zenith angle. As we will detail below, this is compatible with the results presented previously by different authors, and with the flare records included in the GOES database.

In Fig. 1, graphics of $\delta V$ and $\mathrm{d}^{2} \delta V$ in function of time and with a masking angle of $30^{\circ}$ are plotted for stations ascl, crol, kulu and bahr. The overionization is clearly seen by all the satellites in sight of each of those stations. In addition, it can be observed that the sudden increase is produced at almost the same time for the different satellites of each receiver (enhancements are overlapped in each panel), and almost at the same time for the different receivers as well. Furthermore, it is also seen that the amplitude of both $\delta \mathrm{V}$ and $\mathrm{d}^{2} \delta V$ is not equal between different receivers. In other words, the ionospheric impulsive response to solar flares depends on the latitude and longitude of the
Ionospheric Pierce Point (IPP). This effect is mostly due to the overionization dependence on the solar zenith angle (i.e., the angle between the local zenith and the direction of the Sun), as represented in Fig. 3. In this context, it can be observed that while the solar zenith angle from the IPP decreases, the $\delta V$ increases. That was previously expected because there is less ionization efficiency when the Sun is near dawn or dusk.

Another important result is that the TEC increase is detected in all the receivers located in the sunlit region. This area has been roughly represented in Fig. 2 delimiting dawn and dusk by two vertical lines. Additionally, two stations outside this region detect the flare as well. The reason is that the IPPs of several satellites in sight of those stations are still irradiated by the Sun. In our case, as the height of our model of ionosphere is $450 \mathrm{~km}$, the flare could have been detected in stations on night side as far as $20^{\circ}$ from the solar terminator.

In Fig. 3, $\delta V$ is represented in function of the solar zenith angle. The results show that $\delta V$ becomes higher than 15 TECUs near the maximum solar zenith angle and less than 5 TECUs near horizon. It is also illustrated the low overionization related to stations located outside the sunlit area (see Fig. 2), i.e., with a solar zenith angle greater than $90^{\circ}$.

One of the main points of interest is that the ionospheric TEC response to GPS signals of receivers vesl (3W 72S), syog (40E 69S), riog (68W 54S), kerg (70E 49S) and simo (18E 34S) shows a lower level of TEC enhancement than of other receivers with similar mean solar zenith angle. It is important to emphasize that these stations are located in the southern hemisphere and far from the geomagnetic equator. This effect might be related to a different overionization dynamic.

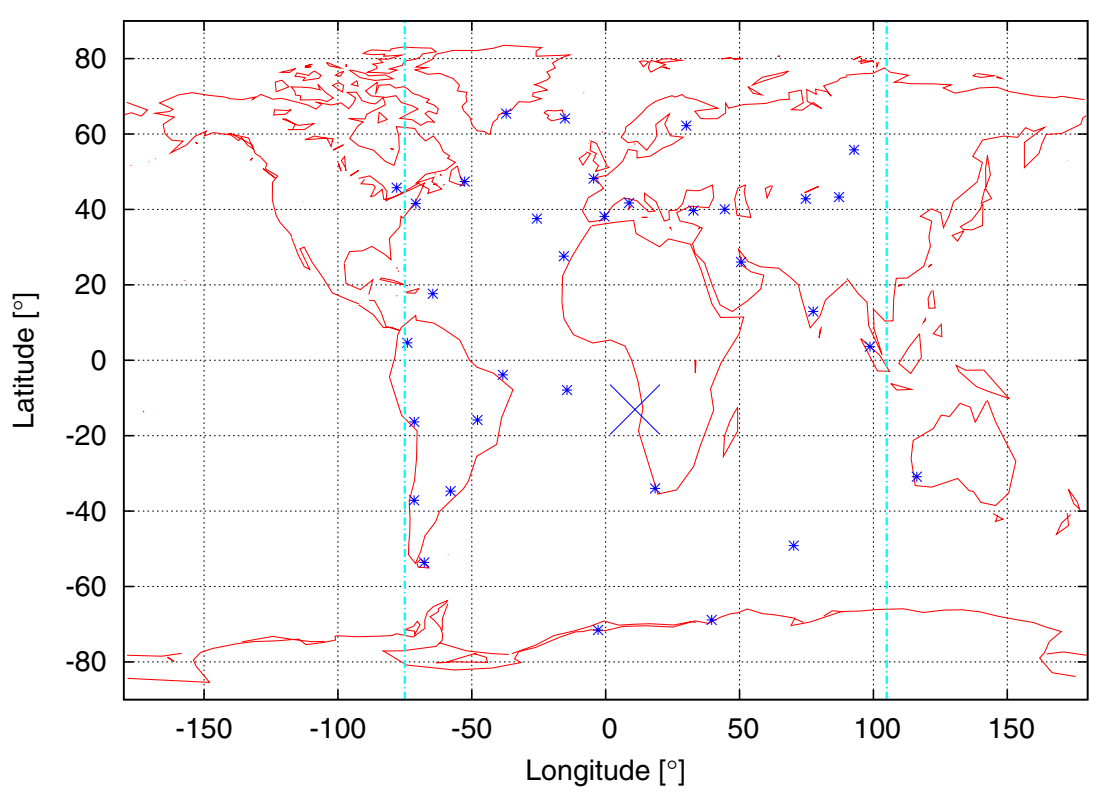

Fig. 2. Distribution of the GPS reference stations that allow the detection of the Halloween storm at about 11 UT, on 28th October, 2003. The large crossmarks the Sun's subsolar point and the two vertical lines roughly delimit the sunlit area. 


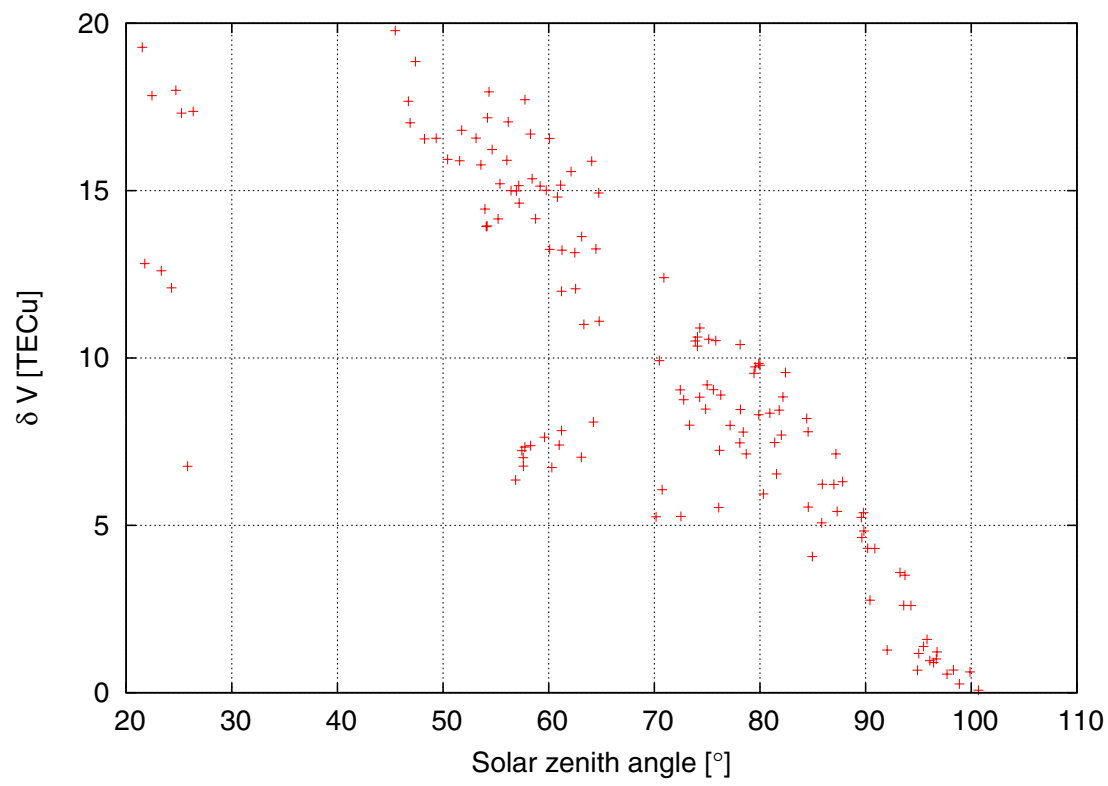

Fig. 3. Dependence of $\delta V$ on the solar zenith angle (from the Ionospheric Pierce Point) for the Halloween storm.

Finally, there are several external comparisons that have been performed to guarantee that the detector works properly. According to GOES, this flare started at 0951 UT and had its peak of intensity at $1110 \mathrm{UT}$, which is compatible with our results. Moreover, they are compatible as well with the ones presented previously in different articles, such as the one presented by Krankowski et al. (2003) and Zhang and Xiao (2005). From another point of view, the $K_{\mathrm{p}}$ geomagnetic index around 28th October, 2003 (day of year 301) is illustrated on Fig. 4. It can be seen clearly that the geomagnetic activity was extremely high 2 days after the radiation arrival. That was previously expected because it was the moment when the particles related to the Halloween storm reached the Earth.

But there are other possible scenarios, such as the solar flare which occurred on 14th July, 2000. This flare has been classified as class X5.7 and is usually referred to as "La Bastille" storm. It took place at the center of the solar disc and the radiation reached the Earth at approximately 1003 UT.

In Fig. 5, a graph of $\delta V$ and $\mathrm{d}^{2} \delta V$ with respect to time is plotted for station bahr. In this figure, it seems that the radiation arrives in three different bursts that are very close to each other, i.e., the slope of the TEC enhancement has minor disturbances contained in it. This implies that the

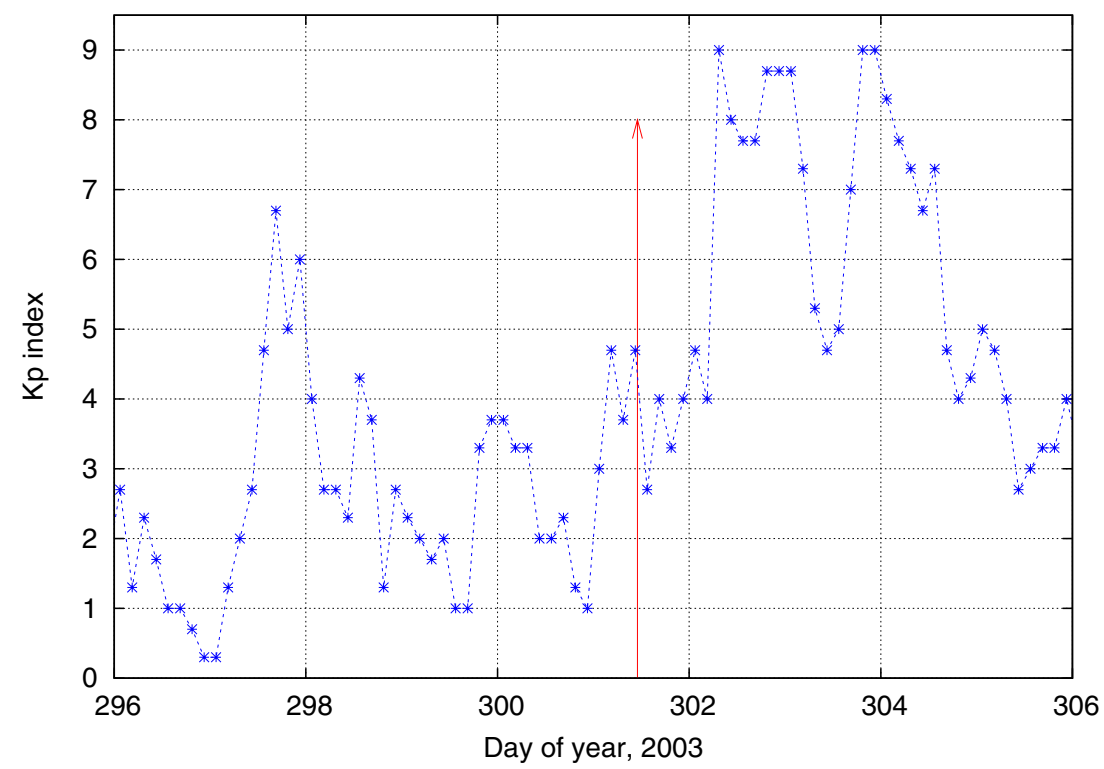

Fig. 4. Variations of $K_{\mathrm{p}}$ index around 28th October, 2003 (day of year 301). The arrow marks the approximate time of the flare event. 


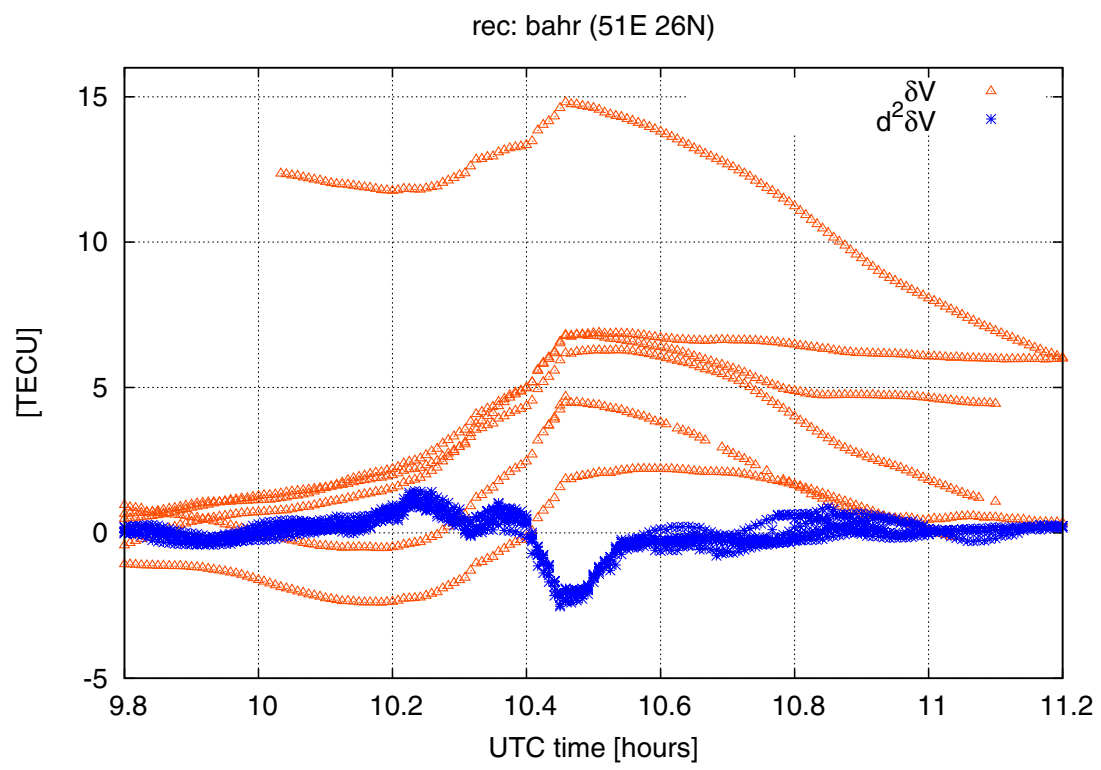

Fig. 5. $\delta V$ and $\mathrm{d}^{2} \delta V$ as function of time obtained from the GPS signals gathered by receiver bahr during "La Bastille" storm and considering a masking angle of $30^{\circ}$.

assumption that a solar flare induces a simple abrupt increase of TEC is not correct. In this context, similar conclusions to this flare have been reached by the authors of Zhang et al. (2002).

Consequently, the typical overionization pattern is not found using the detector based on five states. However, the detector based on a more general criteria, relying on simultaneous observation of just the positive $T E C$ increase in the sunlit region, can detect this flare in spite of the minor TEC disturbances. Further studies can be done to characterize the performance of this more general detector.

It is worth mentioning that the difference of $\delta V$ for one of the satellites in Fig. 5 can be due to the higher electron content gradients associated to the equatorial anomalies.

Finally, a different case of solar flare, of class X7.1 that occurred on the 20th of January 2005, is also analyzed in brief. It is important to emphasize that it was not generated near the center of the solar disc (by GOES results, it was located $14^{\circ}$ North and $61^{\circ}$ West with respect to the center).

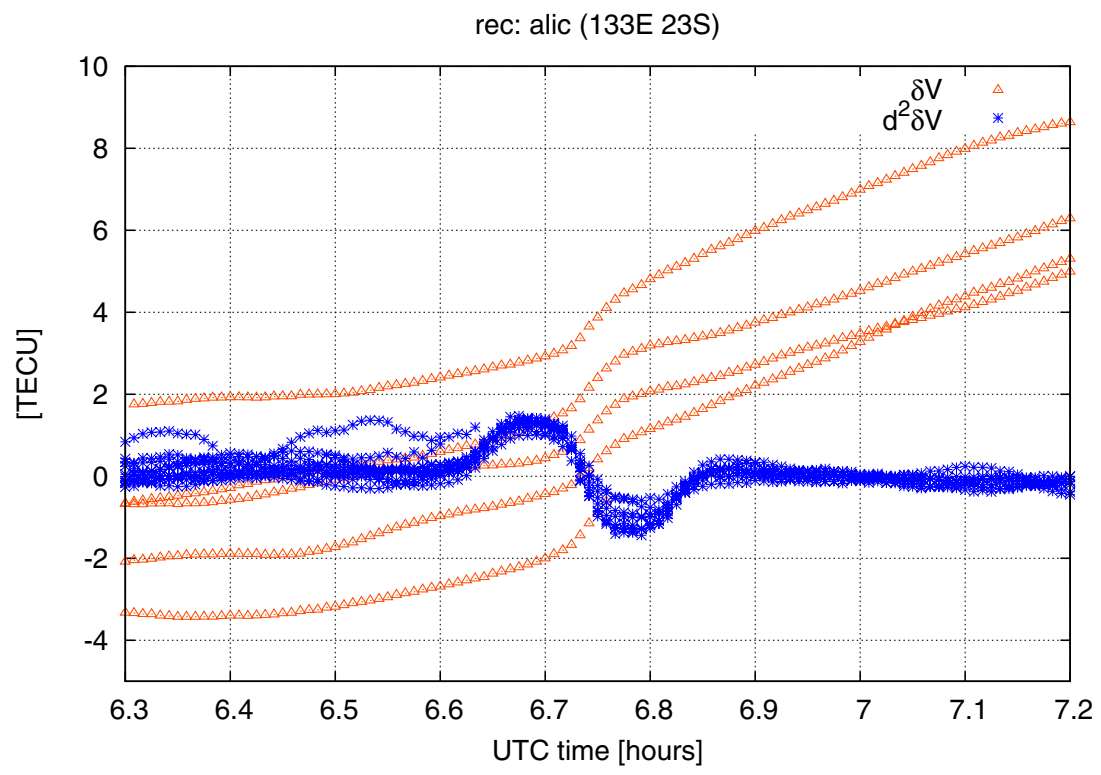

Fig. 6. $\delta V$ and $\mathrm{d}^{2} \delta V$ as function of time obtained from the GPS signals gathered by receiver alic showing the solar storm on 20th January, 2005, and considering a masking angle of $30^{\circ}$. 
Regarding GOES results, the flare started at 0636 UT and had its peak of intensity at 0701 UT. That is compatible with the results obtained in this work (as can be appreciated in Fig. 6).

\section{Conclusions}

The first results of a new solar flare detector, based on the GPS system, have been presented. This detector can automatically identify sudden changes of the ionospheric ionization. Also, it can be applied in real time as well. The detector has been validated with external data such as the X-rays GOES database. In addition, the results have also been compared with the ones presented in recent papers where certain solar storms have been studied using the GPS as well in a more local context, and they are compatible too.

Future work can be focused on: looking for solar flares on large periods of time; statistical measurements between different solar flare ionospheric responses; the improvement of the technique to detect solar flare events and the recombination process after the overionization, among others.

\section{References}

Hernández-Pajares, M., Juan, J.M., Sanz, J., et al. Improving the real time ionospheric determination from GPS sites at very long distances over the equator. J. Geophys. Res. 107 (A10), 1296, doi:10.1029/ 2001JA009203, 2002.

Hernández-Pajares, M., Juan, J.M., Sanz, J. High resolution TEC monitoring method using permanent ground GPS receivers. Geophys. Res. Lett. 24 (13), 1643-1647, 1997.

Krankowski, A., Shagimuratov, I.I., Baran, L.W., et al. Response of the ionosphere to the great solar flare on October 28, 2003. Poster in: European Geosciences Union, General Assembly, 2003.

Liu, J.Y., Tsai, C.H.F., Liou, Y.A. Ionospheric solar flare effects monitored by the ground based GPS receivers: theory and observation. J. Geophys. Res. 109 (A01307), doi:10.1029/2003JA009931, 2004.

Mendillo, M., Klobuchar, J.A., Fritz, R.B. Behavior of the ionospheric F region during the great solar flare of August 7, 1972. J. Geophys. Res. 79, 665-672, 1974.

Zhang, D.H., Xiao, Z. Study of the ionospheric response to the 4B flare on 28 October 2003 using International GPS Service network data. J. Geophys. Res. 110 (A03307), doi:10.1029/2004JA010738, 2005.

Zhang, D.H., Xiao, Z., Igarashi, K., et al. GPS derived ionospheric total electron content response to a solar flare that occurred on 14 July 2000. Radio Sci. 37 (5), 1086, doi:10.1029/2001RS002542, 2002. 